\title{
Survival of patients receiving systematic therapy for metachronous or synchronous metastatic renal cell carcinoma: a retrospective analysis
}

Sung Han Kim', Dong-eun Lee², Boram Park², Jungnam Joo², Jae Young Joung ${ }^{1}$, Ho Kyung Seo', Kang Hyun Lee ${ }^{1}$ and Jinsoo Chung ${ }^{1 *}$

\begin{abstract}
Background: The differences in progression-free survival (PFS) and cancer-specific survival (CSS) of metastatic renal cell carcinoma (mRCC) patients according to treatment, type of metastasis, and Heng criteria risk are unclear. In this study, we compared survival according to various such parameters.

Methods: Between 2000 to 2014, 214 mRCC patients, of whom 171 (79.9\%) were intermediate-risk and 43 (20.1\%) were poor-risk, were retrospectively selected; 126 (58.9\%) patients were treated with immunotherapy (IT) and 88 (41.1\%) with targeted therapy (TT). Moreover, 144 patients had synchronous mRCCs (67.3\%, SM) and 70 had metachronous mRCCs (32.7\%, MM). The Kaplan-Meier method and log-rank test were used to compare progression-free survival (PFS) and CSS.

Results: During a median $4.2(1.0-70.4)$ months of systemic treatment and 98.3 (4.8-147.6) months of followup, the median PFS and CSS were 4.7 (95\% confidence interval [Cl]: 3.8-5.5) and 13.8 (95\% Cl, 9.8-18.3) months, respectively. The PFS and CSS were significantly better in the MM (5.9 and 21.3 months) and intermediate-risk groups (5.2 and 18.3 months) than those in the SM (4.4 and 9.6 months) and poor-risk groups ( 2.7 and 5.8 months), respectively $(p<0.05)$. Further stratification showed that $\Pi$ produced significantly better PFS than IT in intermediate-risk patients with SM and a treatment-free interval (TFI) $<1$ year, and in those with MM with a TFI $\geq 1$ year $(p<0.05)$. There were no differences in survival outcomes according to various other subgroup stratifications $(p>0.05)$.
\end{abstract}

Conclusion: Dividing patients into specific subcategories helps to better predict therapeutic outcomes.

Keywords: Renal cell carcinoma, Metastasis, Synchronous, Metachronous, Prognosis, Immunotherapy, Targeted therapy

\section{Background}

The standard systemic therapy for metastatic renal cell carcinoma (mRCC) has recently been changed to targeted therapy (TT). TT produces improved prognosis, with an observed median cancer-specific survival (CSS) of 29.5 months, and has markedly extended progressionfree survival (PFS) intervals [1]. Furthermore, TT is relatively well tolerated compared to immunotherapy (IT) with cytokines [2], which was the mainstay systemic

\footnotetext{
* Correspondence: cjs5225@ncc.re.kr

'Department of Urology, Center for Prostate Cancer, National Cancer Center, 323 Ilsan-ro, Ilsandong-gu, Goyang-si, Gyeonggi-do 10408, Republic of Korea Full list of author information is available at the end of the article
}

treatment in the past decades [3]. Despite poor 5-year survival rates of approximately $10 \%$, IT had an advantage of significantly improving overall survival (OS), with some patients achieving 10-year persistent complete remission rates of $7-10 \%[4,5]$. Conversely, whether TT prolongs OS has not yet been determined [4]. The heterogeneous characteristics of mRCC patients, as well as the heterotrophic characteristics of their tumors, makes the prediction of therapeutic outcomes challenging. Therefore, dividing patients into subcategories ought to be considered more thoroughly to derive more reliable predictive prognostic factors.

(c) The Author(s). 2019 Open Access This article is distributed under the terms of the Creative Commons Attribution 4.0 International License (http://creativecommons.org/licenses/by/4.0/), which permits unrestricted use, distribution, and reproduction in any medium, provided you give appropriate credit to the original author(s) and the source, provide a link to the Creative Commons license, and indicate if changes were made. The Creative Commons Public Domain Dedication waiver (http://creativecommons.org/publicdomain/zero/1.0/) applies to the data made available in this article, unless otherwise stated. 
mRCCs are categorized into synchronous mRCC (SM), which involves approximately one-third of newly diagnosed patients; and metachronous mRCC, (MM), that has a prevalence rate of $30-40 \%$ among localized RCC patients and involves disease progression to metastasis after a certain interval of time has elapsed since curative surgery. MM and SM have different pathophysiologies and metabolisms; furthermore, the heterotrophic and pleomorphic characteristics of RCC result in unpredictable and diverse responses to systemic therapy. Moreover, the microenvironments and tumor activities of primary vs. metastatic tumor lesions, as well as their underlying tumor burdens, are different $[3,6]$. However, the TT treatment guidelines for MM and SM are similar, and not many studies have addressed the differential prognoses and comparative responses between TT and IT according to the tumors' metastatic types and prognostic risk groups. Such clinical data regarding survival outcomes are required for clinicians to understand the patients' prognoses and to devise effective treatment strategies. Therefore, this retrospective study analyzed the PFS and CSS according to first-line systemic therapy, as well as the survival rates of patients with MM or SM mRCCs treated with either TT or IT at a single institution. Furthermore, prognostic outcomes according to the types of metastases were compared with respect to the Heng risk model and treatment-free intervals (TFIs).

\section{Methods}

\section{Ethics statement}

Following approval of this retrospective study by the Institutional Review Board of the National Cancer Center (IRB No. NCC2016-0263), the IRB waived the written informed consent requirement. All patient data were anonymized and de-identified prior to our analysis. All study protocols were performed in accordance with the ethical tenets of the Declaration of Helsinki.

\section{Patients' criteria and evaluating tools}

The medical records of $214 \mathrm{mRCC}$ patients treated between 2000 and 2014, including 171 (79.9\%) intermediate-risk and $43(20.1 \%)$ poor-risk patients according to the Heng criteria, were retrospectively reviewed. Patients with the following characteristics were excluded from the analysis: those with incomplete follow-up medical records (records beyond the prospectively recorded National Center Center RCC registry), patients who refused systemic therapies after receiving an explanation of the possible adverse events, patients who stopped receiving medications because of the associated financial expenses, patients for whom no therapeutic effects were expected because of poor clinical statuses involving multiple underlying diseases and severe tumor burdens, patients with missing data for all of the risk factors included in the analysis, patients with missing treatment records, patients under 20 years of age, patients belonging to the favorable risk group according to the Heng risk criteria, and patients who developed progression within 1 month of treatment (Additional file 1: Figure S1). There were $144 \mathrm{SM}$ patients $(67.3 \%)$ and 70 MM patients (32.7\%); 126 patients were treated with IT and 88 were treated with TT. All mRCC patients underwent a complete evaluation after every $1-4$ cycles (6-12 weeks) of IT and every 2 cycles of TT (12 weeks). The follow-up protocol that included laboratory and imaging evaluations was previously described in detail [7]. Treatment continued until disease progression was detected. Patients were further stratified into TFI $<1$ year vs. TFI $\geq 1$ year groups. The TFI was defined as the time from the diagnosis of disease to the start of systemic treatment [8-10]

The International Metastatic Renal Cell Carcinoma Database Consortium risk criteria (also known as the Heng criteria) [8] for prognostic risk stratification, the Response Evaluation Criteria in Solid Tumors v1.1 for therapeutic responsive evaluation to systemic therapy [11], and the Fuhrman nuclear grade [12] and TNM stages for pathological RCC evaluation [13] were used.

\section{Treatment regimens}

The choice of systemic agent (IT or TT) was at the discretion of the treating urologist (J.C.) according to each patient's pathology and coverage by the National Health Insurance System, as described previously [7]. Combination IT comprised of subcutaneous recombinant human interleukin (IL)-2 (Proleukin, Chiron V.B.), recombinant human interferon (IFN)- $\alpha$ (IFN-alpha-2a, Roferon-A, Roche), intravenous 5-fluorouracil (5-FU, JW Pharm), and vinblastine (vinblastine, United Pharm, Korea). Triple or quadruple regimens were administered according to our previous cited regimen [14].

All TTs were administered either orally or intravenously with the recommended regimen in the National Comprehensive Cancer Network guidelines, version 2.2016 (available at http://www.nccn.org/patients for patients). First-line TT comprised sunitinib, sorafenib, pazopanib, or temsirolimus; sequential TT included sunitinib, sorafenib, pazopanib, temsirolimus, bevacizumab, everolimus, or axitinib. Targeted-agent regimens were described previously $[7,15]$.

\section{Statistical analysis}

The baseline characteristics are summarized as frequency with percentage for categorical variables and median with range (min-max) for continuous variables. The differences between SM and MM were assessed using the Wilcoxon rank-sum test and chi-square test (or Fisher's exact test). The date of the most recent hospital visit 
was used as the date of last follow-up. The PFS duration was defined as the time between first-line systemic therapy and disease progression. The CSS duration was defined as the time between first-line systemic therapy and death from cancer or alive. Follow-up durations were estimated using the reverse Kaplan-Meier method, in which being alive is treated as the event of interest and deaths are censored. The estimated survival curves were computed using the Kaplan-Meier method. Survival curves according to systemic treatment and Heng risk groups were compared using the log-rank test. Cox proportional hazards models were used to analyze subgroups of the patients with mRCC. To identify factors associated with prognosis, univariable Cox models of PFS and CSS were applied to the variables included in the Heng risk model. Variables that had significant $(p<$ 0.05) associations with PFS or CSS in the univariable analyses were included in multivariable Cox models. A backward variable selection method was then applied to the included variables with an elimination $p$-value criterion of 0.05 . $P$-values less than 0.05 were considered statistically significant. All statistical analyses were performed using SAS (version 9.3; SAS Institute Inc., Cary, NC, USA) and R (version 3.3.2) software.

\section{Results}

Following a median 4.2 (range: $1.0-70.4$ ) months of systemic treatment and 98.3 (range: 4.8-147.6) months of follow-up, only 30 patients $(14.0 \%)$ were alive at the end of the study. The median PFS and CSS were 4.7 (95\% confidence interval: $3.8-5.5)$ months and 13.8 (95\% confidence interval: 9.8-18.3) months, respectively. The patients' characteristics are summarized in Table 1 . The gender ratio, nephrectomy rates, TFIs, Heng risk groups, clinical $\mathrm{T}$ and $\mathrm{N}$ stages, clear cell and nonclear cell histologies, follow-up durations, PFS, and CSS were significantly different between the SM and MM groups $(p<0.05$, Table 2$)$.

Significantly better PFS and CSS rates in the MM group (PFS: 5.9 months; CSS: 21.3 months) and in the intermediate-risk group (PFS: 5.2 months; CSS: 18.3 months) were observed than in the SM (PFS: 4.4 months; CSS: 9.6 months) and poor-risk groups (PFS: 2.7 month; CSS: 5.8 months) $(p<0.05$; Additional file 1 : Figure S2). In the intermediate-risk groups, patients with MM showed longer PFS and CSS rates than those with SM (PFS: 6.2 vs. 5.1 months; CSS: 25.2 vs. 13.9 months, respectively). Furthermore, the MM group showed longer PFS and CSS than the SM group (3.7 vs. 2.7 months and 10.2 vs. 5.6 months, respectively) in poor-risk patients (Additional file 1: Figure S2); statistical significance was only achieved for CSS in the intermediate-risk group ( $p=0.01$ ) (Additional file 1: Figure S3).
Among patients with SM, those receiving TT had significantly longer PFS (5.2 months) than those receiving IT (2.7 months, $p<0.001$; Fig. 1a). Similarly, among patients with MM, those receiving TT (9.7 months) had significantly longer PFS than those receiving IT (4.1 months, $p=0.006$; Fig. 1c). However, CSS did not differ significantly between SM patients receiving IT (9.1 months) and those receiving TT (9.6 months; $p=0.472$; Fig. 1b), or between MM patients receiving IT (25.2 months) and those receiving TT (20.1 months; $p=0.229$; Fig. 1d).

As for the stratified Heng risk groups, only the intermediate-risk group treated with TT had a significantly longer PFS (6.1 months) than that of the IT group (2.6 months) among SM patients $(p<0.001$, Fig. $2 \mathrm{a})$, although CSS was not significantly different $(p=0.67$; Fig. $2 \mathrm{~b})$. There were no differences in PFS and CSS when comparing IT vs. TT among poor-risk SM patients ( $p=$ 0.164 for PFS, $p=0.083$ for CSS; Fig. 2c-d). TT significantly improved PFS (TT: 10.2 months; IT: 4.1 months, $p=0.004$; Fig. 2e) but not CSS among patients with MM in the intermediate-risk group $(p=0.262$; Fig. 2f), whereas PFS and CSS were not significantly different when comparing IT vs. TT among poor-risk MM patients ( $p=0.863$ for PFS, $p=0.352$ for CSS; Fig. $2 g-h$ ).

When stratifying patients according to TFI of $<1$ year vs. $\geq 1$ year, intermediate-risk patients treated with TT had significantly better PFS than those treated with IT (6.1 months vs. 2.6 months, respectively) among SM patients with TFI $<1$ year $(p<0.001$, Fig. 3a). However, no other significant differences in PFS or CSS were observed between IT- and TT-treated patients with SM or MM and TFI $<1$ year $(p>0.05$, Fig. 3$)$. Among patients with $\mathrm{mRCC}$ and TFIs $\geq 1$ year, only intermediate-risk MM patients treated with TT had significantly better PFS (10.5 months) than those treated with IT (4.0 months) ( $p=0.008$; Fig. $4 \mathrm{c}$ ). The poor-risk patients with TFIs $\geq 1$ year could not be assessed because of their small sample size.

The results of univariable and multivariable Cox proportional hazards analyses of PFS and CSS are summarized in Additional file 1: Table S1. In the univariable analyses of PFS, metastatic type, therapy, TFI, neutrophilia, and elevated LDH were statistically significant prognostic factors. In the multivariable analysis of PFS, therapy (hazard ratio [HR]: 0.537; 95\% CI: 0.399-0.724; $p<0.001$ ), TFI (HR: 1.639; 95\% CI: 1.171-2.293; $p=0.004)$, and neutrophilia (HR: 1.695 ; 95\% CI: $1.141-2.516 ; p=0.009$ ) were statistically significant factors. In the univariable analyses of CSS, metastatic type, TFI, hypercalcemia, neutrophilia, elevated $\mathrm{LDH}$, and thrombocytopenia were significant prognostic factors. In the multivariable analysis of CSS, TFI (HR: 2.115; 95\% CI: 1.484-3.015; $p<0.001)$, hypercalcemia (HR: 1.788; 95\% CI: 1.163-2.748; $p=0.008$ ), neutrophilia 
Table 1 Baseline characteristics table $(N=214)$

\begin{tabular}{|c|c|c|}
\hline & & $\begin{array}{l}\mathrm{N}(\%) \text { or Median } \\
\text { (min-max) }\end{array}$ \\
\hline Age (years) & & $58(26-81)$ \\
\hline \multirow[t]{2}{*}{ Gender } & Male & $166(77.6)$ \\
\hline & Female & $48(22.4)$ \\
\hline \multirow[t]{3}{*}{ Nephrectomy } & No & $90(42.1)$ \\
\hline & Yes & $121(56.5)$ \\
\hline & Unknown & $3(1.4)$ \\
\hline \multirow[t]{2}{*}{ Treatment free interval } & $\geq 1 \mathrm{yr}$ & $56(26.2)$ \\
\hline & $<1 \mathrm{yr}$ & $158(73.8)$ \\
\hline \multirow[t]{2}{*}{ Anemia } & Normal & $44(20.6)$ \\
\hline & $\mathrm{Hb}<13.5 / 12.0(\mathrm{M} / \mathrm{F})$ & $170(79.4)$ \\
\hline \multirow[t]{3}{*}{ Hypercalcemia } & Normal & $180(84.1)$ \\
\hline & $\begin{array}{l}>10 \mathrm{mg} / \mathrm{dL} \text { or } \\
2.5 \mathrm{mmol} / \mathrm{L}\end{array}$ & $28(13.1)$ \\
\hline & Unknown & $6(2.8)$ \\
\hline \multirow[t]{3}{*}{ Neutrophilia } & Normal & $174(81.3)$ \\
\hline & $<1500$ or $>7500$ & $33(15.4)$ \\
\hline & Unknown & $7(3.3)$ \\
\hline \multirow[t]{3}{*}{ Elevated LDH } & Normal & $96(44.9)$ \\
\hline & $>\times 1.5 \cup L N$ & $49(22.9)$ \\
\hline & unknown & $69(32.2)$ \\
\hline \multirow[t]{3}{*}{ KPS } & $>80$ & $204(95.3)$ \\
\hline & $\leq 80$ & $4(1.9)$ \\
\hline & unknown & $6(2.8)$ \\
\hline \multirow[t]{2}{*}{ Thrombocytosis } & Normal & $192(89.7)$ \\
\hline & $>400 \mathrm{~K}$ & $22(10.3)$ \\
\hline \multirow[t]{2}{*}{ Therapy } & Immunotherapy & $126(58.9)$ \\
\hline & Target Therapy & $88(41.1)$ \\
\hline \multirow[t]{2}{*}{ Heng } & Intermediate risk & $171(79.9)$ \\
\hline & Poor risk & $43(20.1)$ \\
\hline \multirow[t]{3}{*}{ Tumor $(T)$} & $\mathrm{T} 1-\mathrm{T} 2$ & $96(44.9)$ \\
\hline & $\mathrm{T} 3-\mathrm{T} 4$ & $61(28.5)$ \\
\hline & Unknown & $57(26.6)$ \\
\hline \multirow[t]{4}{*}{ Lymph node(N) } & NO & $54(25.2)$ \\
\hline & N1 & $45(21.0)$ \\
\hline & $\mathrm{Nx}$ & $30(14.0)$ \\
\hline & Unknown & $85(39.7)$ \\
\hline \multirow[t]{5}{*}{ Metastasis } & $\mathrm{cMO}$ & 27 (12.6) \\
\hline & cM1 & 81 (37.9) \\
\hline & pM1 & $2(0.9)$ \\
\hline & $c M x$ & $3(1.4)$ \\
\hline & Unknown & $101(47.2)$ \\
\hline \multirow[t]{2}{*}{ mRCC type } & $\begin{array}{l}\text { Synchronous } \\
\text { mRCC }\end{array}$ & $144(67.3)$ \\
\hline & Metachronous & $70(32.7)$ \\
\hline
\end{tabular}

Table 1 Baseline characteristics table $(N=214)$ (Continued)

\begin{tabular}{|c|c|c|}
\hline & & $\begin{array}{l}\mathrm{N}(\%) \text { or Median } \\
\text { (min-max) }\end{array}$ \\
\hline & $\mathrm{mRCC}$ & \\
\hline \multirow[t]{3}{*}{ Fuhrman nuclear grade } & G1-G2 & $41(19.2)$ \\
\hline & G3-G4 & $101(47.2)$ \\
\hline & Unknown & 72 (33.6) \\
\hline \multirow[t]{3}{*}{ Histology } & Clear cell & $164(76.6)$ \\
\hline & Non-clear cell & $12(5.6)$ \\
\hline & Unknown & $38(17.8)$ \\
\hline \multicolumn{2}{|c|}{ Treatment duration (Month) } & $4.2(1.0-70.4)$ \\
\hline \multicolumn{2}{|c|}{ Follow-up duration (Month) } & $98.3(4.8-147.6)$ \\
\hline \multicolumn{2}{|c|}{ Progression free survival (Month, median $(95 \% \mathrm{Cl})$ ) } & $4.7(3.8-5.5)$ \\
\hline \multicolumn{2}{|c|}{ Cancer-specific survival (Month, median(95\%Cl)) } & $13.8(9.8-18.3)$ \\
\hline $\begin{array}{l}\text { Cancer-specific survival } \\
\text { status }\end{array}$ & Censored/ Event & $32(15.0) / 182(85.0)$ \\
\hline $\begin{array}{l}\text { Progression-free survival } \\
\text { status }\end{array}$ & Censored/ Event & $21(9.8) / 193(90.1)$ \\
\hline
\end{tabular}

(HR: 1.916 ; 95\% CI: $1.247-2.942 ; p=0.003$ ), and thrombocytosis (HR: 1.856; 95\% CI: 1.092-3.157; $p=0.022$ ) were significant factors.

Cox analyses of PFS for the SM and MM subgroups are shown in Table 3. In univariable analyses of the SM subgroup, Heng risk classification, therapy, anemia, neutrophilia, and thrombocytopenia were significant prognostic factors. In the multivariable analysis of the SM subgroup, therapy (HR: 1.696; 95\% CI: 1.157-2.486; $p=$ 0.0068 ) and neutrophilia (HR: 2.105 ; $95 \%$ CI: $1.274-$ 3.478; $p=0.0037$ ) were significant factors. In univariable analyses of the MM subgroup, therapy was the only significant factor.

Cox analyses of CSS in the SM and MM subgroups are shown in Table 4. In univariable analyses of the SM subgroup, Heng risk classification, hypercalcemia, neutrophilia, and thrombocytopenia were significant prognostic factors. In the multivariable analysis of the SM subgroup, hypercalcemia (HR: 2.164; 95\% CI: 1.3073.584; $p=0.0027$ ) and neutrophilia (HR: 2.655 ; $95 \% \mathrm{CI}$ : $1.601-4.405 ; p=0.0002)$ were significant factors. In univariable analyses of the MM subgroup, Heng risk classification and TFI were significant factors.

\section{Discussion}

TT has recently replaced IT as the mainstay therapy for mRCC, resulting in modest survival benefits with improved PFS despite failing to provide measurable benefits to CSS except in poor-risk patients administered temsirolimus [4], as in the present study, where the CSS were the same between poor- and intermediate-risk mRCC. Our previously published study also showed that TT improved prognoses of mRCC patients compared to 
Table 2 Comparison of baseline characteristics between synchronous ( $N=144)$ and metachronous $(N=70)$ mRCC groups

\begin{tabular}{|c|c|c|c|c|}
\hline & & Synchronous & Metachronous & $P$-value \\
\hline & & $(N=144)$ & $(N=70)$ & \\
\hline Age (years, min-max) & & $59.0(26.0-81.0)$ & $56.0(33.0-76.0)$ & $0.232^{a}$ \\
\hline Gender & Male & $118(81.9)$ & $48(68.6)$ & $0.028^{b}$ \\
\hline & Female & $26(18.1)$ & $22(31.4)$ & \\
\hline Nephrectomy & Yes & $52(36.6)$ & $69(100.0)$ & $<.001^{\mathrm{b}}$ \\
\hline Treatment free interval & $\geq 1 \mathrm{yr}$ & $8(5.6)$ & $48(68.6)$ & $<.001^{\mathrm{c}}$ \\
\hline & $<1 \mathrm{yr}$ & $136(94.4)$ & $22(31.4)$ & \\
\hline Anemia & & $111(77.1)$ & $59(84.3)$ & $0.221^{b}$ \\
\hline Hypercalcemia & & $20(14.4)$ & $8(11.6)$ & $0.578^{\mathrm{b}}$ \\
\hline Neutrophilia & & $21(15.1)$ & $12(17.7)$ & $0.639^{b}$ \\
\hline Elevated LDH & $>\times 1.5$ ULN & $39(38.2)$ & $10(23.3)$ & $0.082^{b}$ \\
\hline KPS & $>80$ & $135(97.1)$ & $69(100)$ & $0.304^{c}$ \\
\hline & $\leq 80$ & $4(2.9)$ & $0(0)$ & \\
\hline Thrombocytosis & $>400 \mathrm{~K}$ & $18(12.5)$ & $4(5.7)$ & $0.125^{\mathrm{b}}$ \\
\hline First line therapy & Immunotherapy & $89(61.8)$ & $37(52.9)$ & $0.212^{b}$ \\
\hline & Targeted therapy & $55(38.2)$ & $33(47.1)$ & \\
\hline Heng risk & Intermediate & $107(74.3)$ & $64(91.4)$ & $0.003^{b}$ \\
\hline & Poor & $37(25.7)$ & $6(8.6)$ & \\
\hline Tumor (T) & $\mathrm{T} 1-\mathrm{T} 2$ & $70(67.3)$ & $26(49.1)$ & $0.027^{b}$ \\
\hline & $\mathrm{T} 3-\mathrm{T} 4$ & $34(32.7)$ & $27(50.9)$ & \\
\hline Lymph node(N) & NO & $49(47.1)$ & $5(20.0)$ & $<.001^{\mathrm{b}}$ \\
\hline & N1 & $25(24.0)$ & $20(80.0)$ & \\
\hline & $\mathrm{Nx}$ & $30(28.9)$ & $0(0.0)$ & \\
\hline Fuhrman nuclear grade & G1-G2 & $30(33)$ & $11(21.6)$ & $0.151^{b}$ \\
\hline & G3-G4 & $61(67)$ & $40(78.4)$ & \\
\hline Histology & Clear cell & $113(96.6)$ & $51(86.4)$ & $0.022^{c}$ \\
\hline & Non-clear cell & $4(3.4)$ & $8(13.6)$ & \\
\hline Treatment duration (Mo & & $3.4(1.0-70.4)$ & $5.3(1.0-62.0)$ & $0.058^{\mathrm{a}}$ \\
\hline Follow-up duration (Mo & & $81.3(4.8-141.5)$ & $142.3(9.4-147.6)$ & $0.005^{d}$ \\
\hline Progression free survival & $95 \% C l))$ & $4.4(3.1-5.2)$ & $5.9(4.1-9.5)$ & $0.044^{d}$ \\
\hline Cancer specific survival & $5 \%(\mathrm{Cl}))$ & $9.6(7.4-13.8)$ & $21.3(15.1-29.3)$ & $<.001^{d}$ \\
\hline
\end{tabular}

KPS Karnofsky performance status

${ }^{a}$ Wilcoxon rank sum test, ${ }^{b}$ Chi-square test, ${ }^{c}$ Fisher exact test, d: Log-rank test

IT [7]. In this study, we further stratified mRCC patients in terms of metastasis type, Heng risk model, and TFI. The intermediate and poor risk patients were selected in this study; however, patients belonging to the favorable risk group were excluded because of the characteristics of SM, for which no cases belong to the favorable risk group.

The results of our study showed that the intermediaterisk group had significantly better PFS and CSS than the poor-risk group. We also observed that, as compared with IT, TT significantly lengthened PFS, but not CSS (Fig. 1). In particular, a significant difference in CSS was observed in neither the SM subgroup (TT vs. IT: 9.6 vs.
9.1 months) nor the MM subgroup (TT vs IT: 20.1 vs. 25.2 months; $p>0.05$; Fig. $1 \mathrm{~b}$ and d). Nonetheless, our results provide some statistically nonsignificant indications that IT may be associated with better CSS than TT: in both the SM and MM subgroups (Fig. 1b and d), comparison of the tails of the IT and TT curves for CSS show that the former had longer durable responses without cancer-specific deaths. Overall, our results suggest that TT was significantly better for PFS, while IT might be more suitable for extending CSS. However, the survival benefit from IT should be considered carefully, because the IT group included patients who benefited from TT $(9.5 \%, N=12)$, and their follow-up periods were 


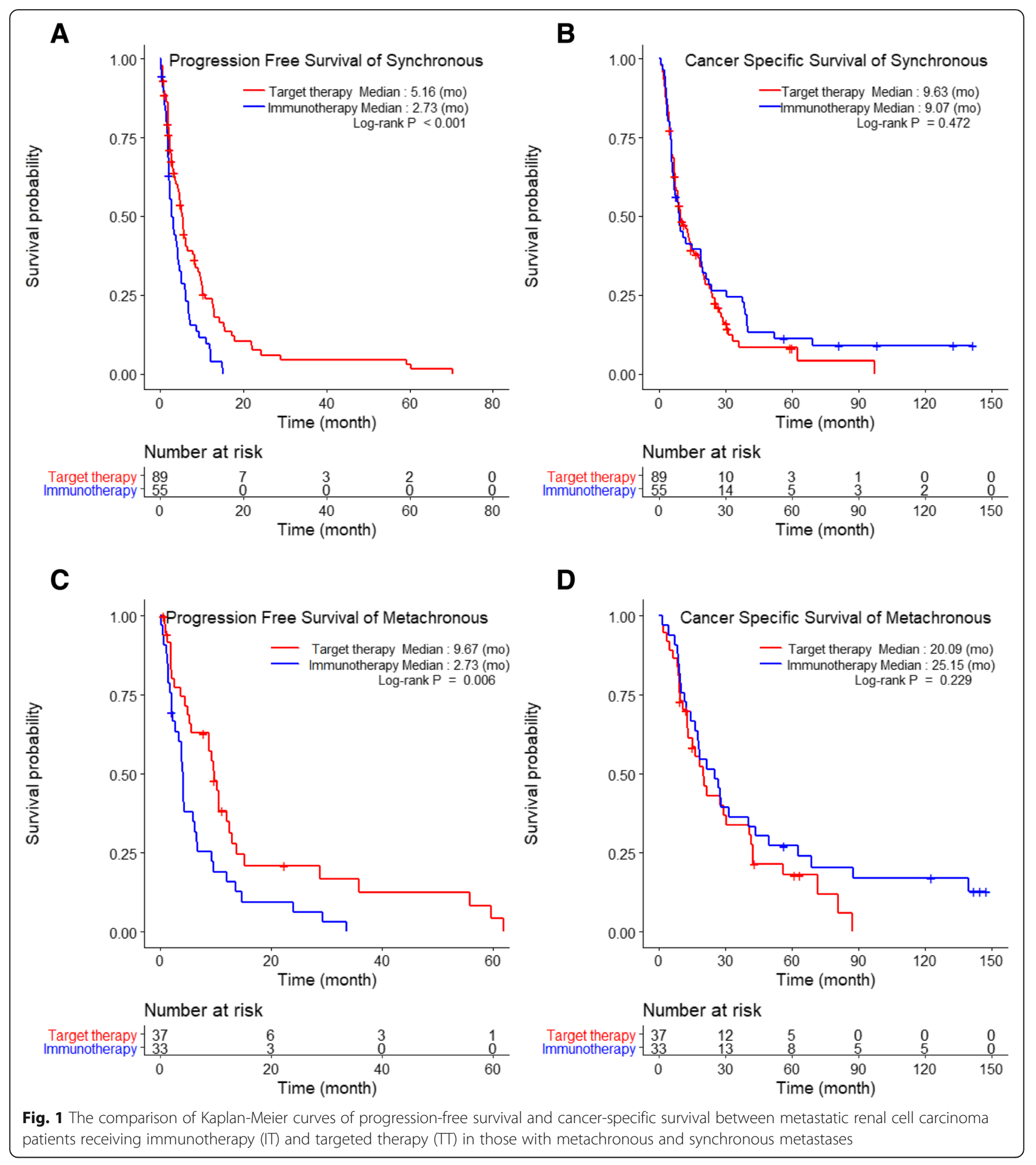

significantly longer than those of TT patients (data not shown). Finally, only SM patients with TFIs $<1$ year and MM patients with TFIs $\geq 1$ year had significantly prolonged PFS when treated with TT compared to IT in the intermediate-risk group. Other subgroups did not show any significant survival differences according to treatment.
In previous large-scale IT and TT studies, Motzer et al. [16] and Naito et al. [17] showed median CSS of 10.0 and 21.5 months following cytokine treatment, respectively; the survival times were 14.0 and 29.5 months for their intermediate-risk (according to the Memorial Sloan Kettering Cancer Center [MSKCC] criteria] groups, and 5.0 and 9.8 months for their MSKCC poor-risk groups, 
A

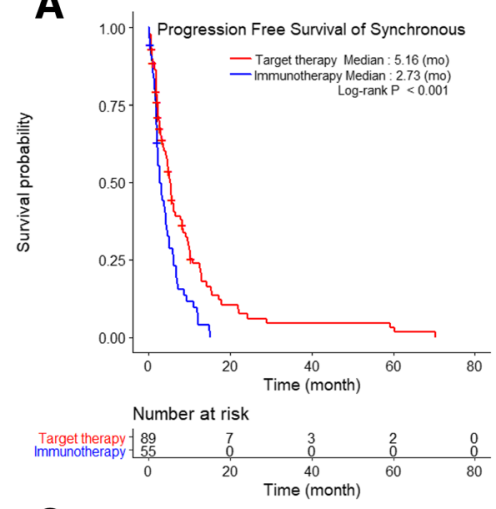

C
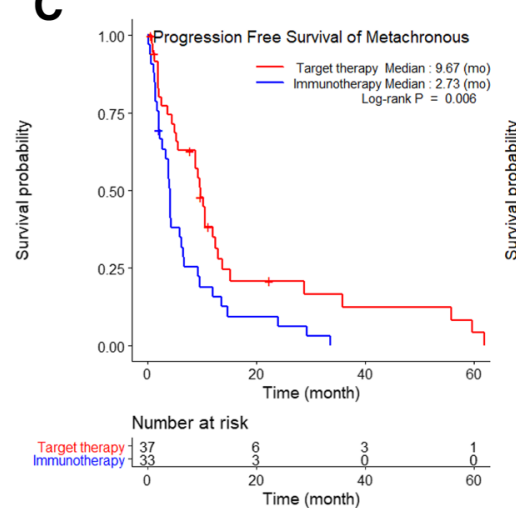

E
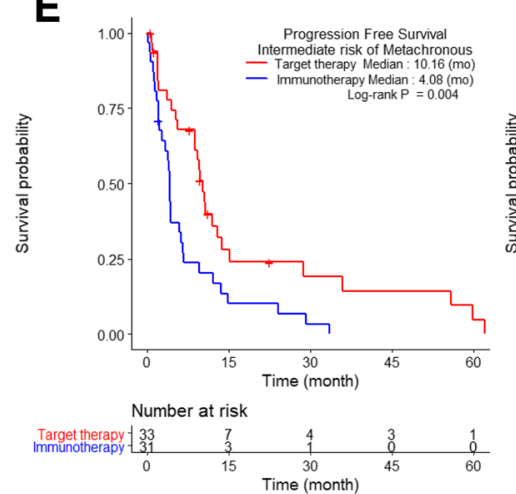

G

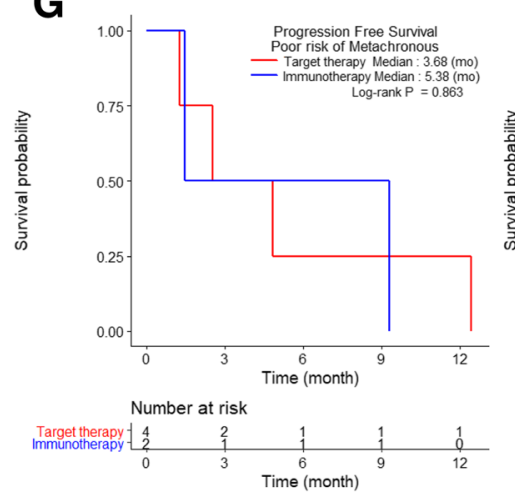

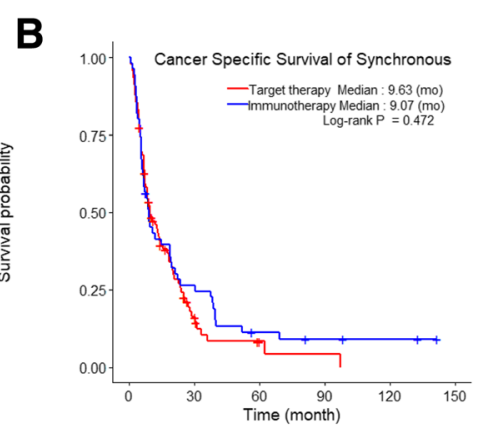

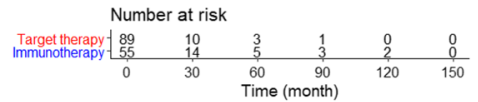

D

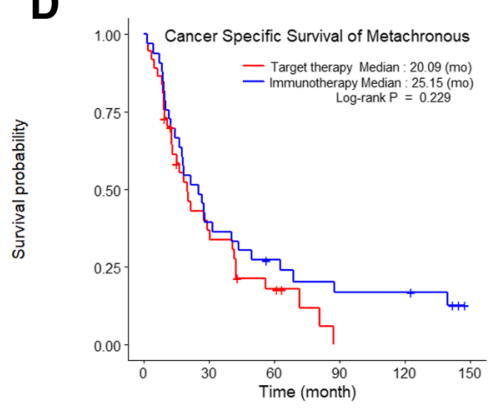

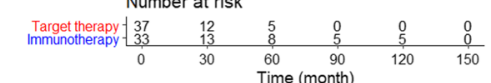

$\mathbf{F}$

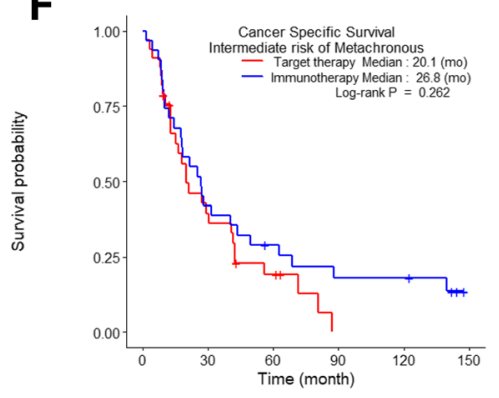

Number at risk

\section{H}

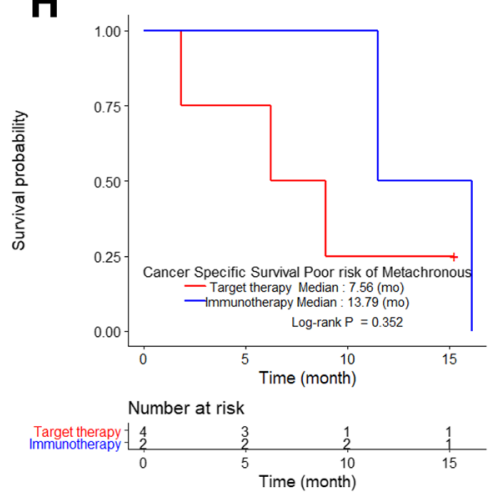

Fig. 2 Comparison of Kaplan-Meier curves of progression-free survival and cancer-specific survival of metastatic renal cell carcinoma patients receiving immunotherapy (IT) and targeted therapy (TT) in groups with synchronous metastases (a-d) and metachronous metastases (e-h) according to the Heng risk groups 



Fig. 3 Comparison of Kaplan-Meier curves of progression-free survival and cancer-specific survival of metastatic renal cell carcinoma patients with treatment-free intervals $<1$ year and with synchronous metastases $(\mathbf{a}-\mathbf{d})$ and metachronous metastases $(\mathbf{e}-\mathbf{f})$ between patients receiving immunotherapy (IT) and targeted therapy (TT) according to the Heng risk groups 


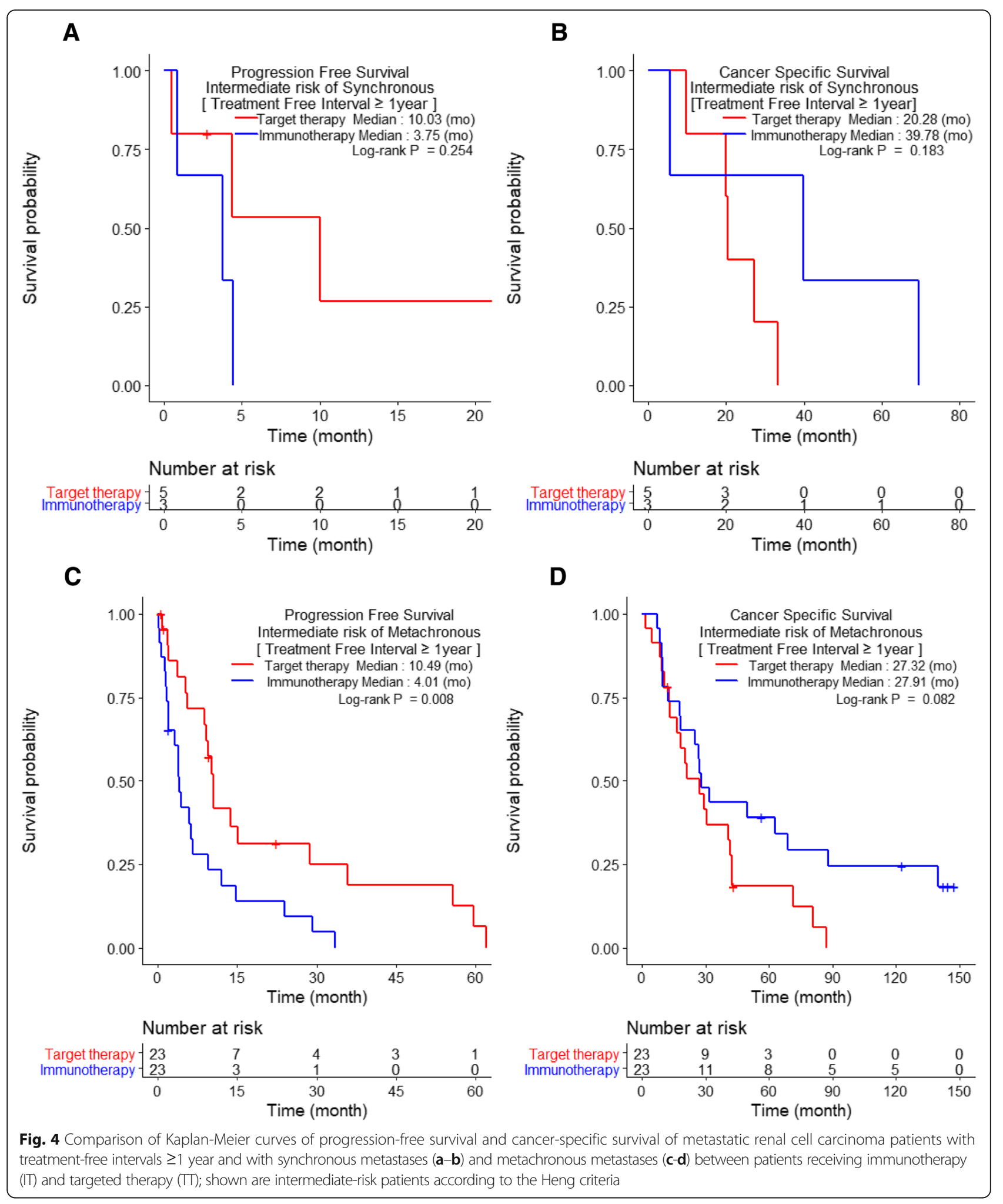

respectively [18]. Our study had similar median CSS results, with respectively 13.9 and 25.2 months for the intermediate-risk and 5.6 and 10.2 months for the poorrisk $\mathrm{SM}$ and $\mathrm{MM}$ groups. Some differences were expected owing to the enrolled patients' ethnic group, rate of nephrectomy (Motzer: 80.5\%, Naito: 55\%, and our study: $56.5 \%$ ), and the different cytokine-treatment regimens and primary and metastatic tumor burdens. 


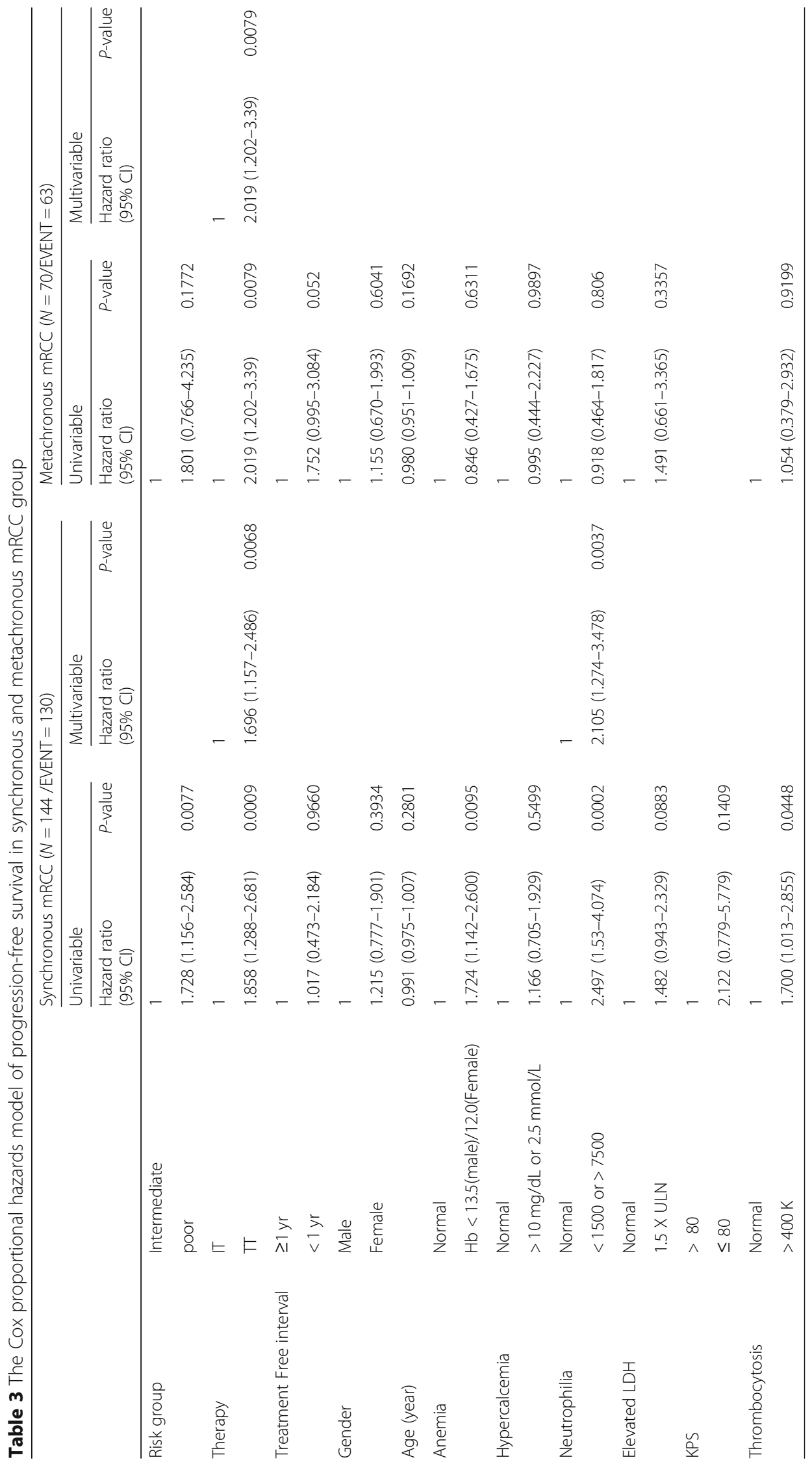




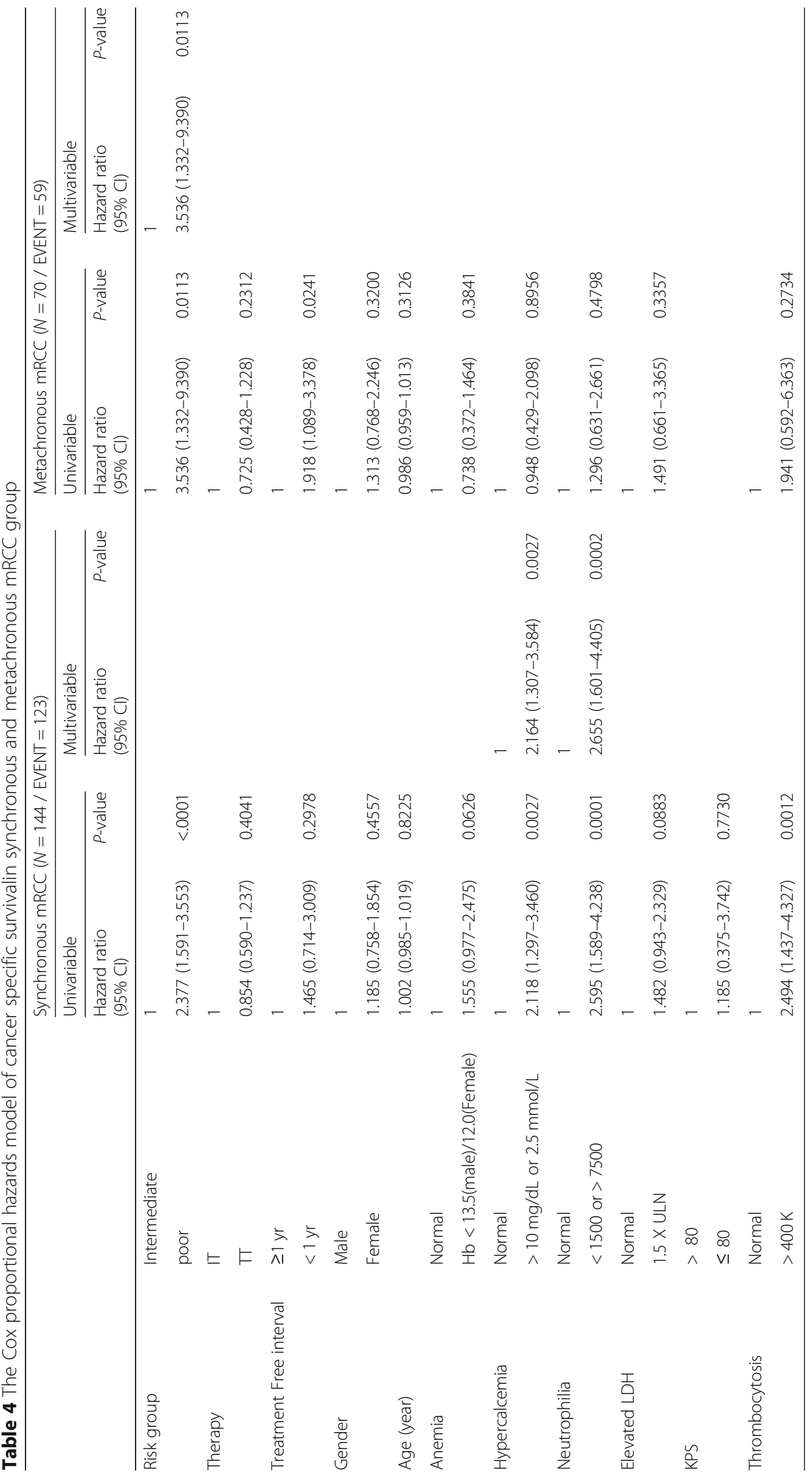


The outcomes of TT in our study were similar to, or slightly poorer than, previous phase 3 trials $[7,14]$. The different outcomes between this study and previous studies were attributed to the different characteristics of the enrolled patients, including the absence of favorablerisk groups in our study.

To our knowledge, no studies have directly compared prognostic outcomes according to metastasis type outside of metastatic site-specific comparison studies and case reports [19-24]. Although tumor cells' pathophysiologies, activities, and burdens differ between SM and MM, the systemic treatment protocol for these cancers remain the same. Previous studies and ours showed different pathophysiologic activities of tumor cells as identified by immunohistochemical staining of multiple tissue markers that are closely related to the TT and immune responses $[15,19]$. The absence of a primary renal tumor burden post-nephrectomy influences prognoses following both IT and TT $[25,26]$.

Our study classified mRCC patients according to the Heng risk criteria, TFI, metastatic types, and systemic therapies to determine the influence of each on prognoses. Prognostic comparisons according to the Heng risk groups showed a significant difference in CSS between SM (13.9 months) and MM (25.2 months) in the intermediate-risk group $(p=0.010)$, but not in PFS. The poor-risk group also showed no prognostic differences according to metastatic types. CSS rates were better in MM than in SM patients because the metastatic tumor burden was less in the former, and the tumor metabolic activity was different from those with SM. When systemic therapies were compared, only the PFS in the intermediate-risk group showed a significant improvement with TT over IT in both the SM (6.1 vs. 2.6 months) and MM (10.2 vs. 4.1 months) groups, respectively. One interesting finding was that receipt of IT was associated with nonsignificantly better PFS and CSS rates for poor-risk patients in both the SM and MM subgroups ( $p>0.05$, Fig. $1 \mathrm{c}$ and $\mathrm{d})$. When considering TFI, TT significantly improved PFS in SM group patients with TFIs < 1 year and in MM group patients with TFIs $\geq 1$ year; these findings were true regardless of metastatic types. In addition, because of multicollinearity between TFI and metastatic type (SM vs. MM), metastatic type was not a significant prognostic factor in the multivariable analysis, even though it had been significantly prognostic in the univariable analysis.

This study had some inherent limitations owing to its retrospective design, the small number of poor-risk patients, the exclusion of patients with medical records that were incomplete because of loss to follow-up, and the different follow-up periods in the compared subgroups. Most metastatic lesions that were diagnosed pathologically might not accurately represent the whole disease, especially when comparing the primary and various metastatic lesions. Further studies that include tissue analysis, gene sequencing, and clinicopathological data are warranted to identify the therapeutic benefit of systemic therapies on MM and SM patients. Prospective data collection and different statistical analyses may help to address the possibility that selection bias and other biases could have affected the comparisons that were made in the present study. For example, the use of propensity score matching might allow more rigorous comparisons of outcomes after IT and TT, as well as of the prognostic implications of SM and MM mRCC. There was a difficulty in applying propensity score matching in this study. Since some of the variables were unknown, the size of the after matching set will be smaller. It is difficult to analyze each sub-groups (SM/MM, risk group, Treatment interval) after matching because IT and TT survival is identified in all subgroups of patients. Our study is only the second to compare prognoses of SM and MM patients treated with systemic TTs.

\section{Conclusions}

Our study showed that dividing patients into additional subcategories improved the prediction of therapeutic outcomes. We found that patients with intermediate-risk mRCC treated with TT had significantly better PFS rates than those treated with IT among patients with SM and TFIs $<1$ year and patients with MM with TFIs $\geq 1$ year.

\section{Additional files}

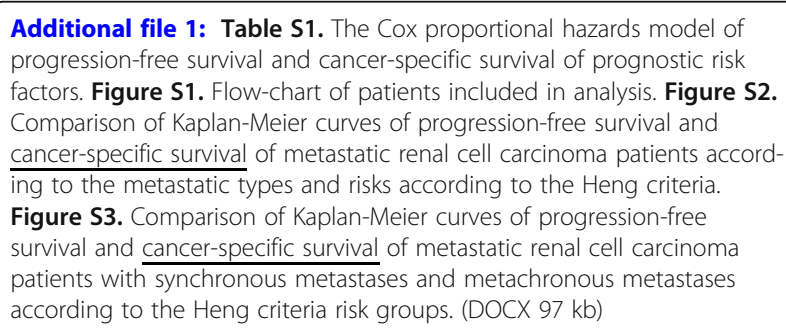

\section{Abbreviations}

(m)RCC: (metastatic) renal cell carcinoma; CSS: Cancer-specific survival; FU: Fluorouracil; IFN: Interferon; IL: Interleukin; IT: Immunotherapy; MM: Metachronous mRCC; OS: Overall survival; PFS: Progression-free survival; SM: Synchronous mRCC; TFI: Treatment-free interval; TT: Targeted therapy

\section{Acknowledgements}

Not applicable.

\section{Authors' contributions}

SHK collected the data and wrote the manuscript. DL, BP, and JJ performed the statistical analysis. JC designed the study, selected the study methodology that was actually used, participated in data collection, and reviewed the manuscript. JYJ, HKS, and KHL participated in data collection and helped to draft the manuscript. All authors read and approved the final manuscript.

Funding

This study was not funded by any external sources. 


\section{Availability of data and materials}

All data generated or analyzed during this study are included in this published article [and its supplementary information files].

\section{Ethics approval and consent to participate}

The Institutional Review Board of the National Cancer Center approved this study (IRB No. NCC2016-0263). The IRB waived the written informed consent requirement.

\section{Consent for publication}

Not applicable.

\section{Competing interests}

The authors declare that they have no competing interests.

\section{Author details}

'Department of Urology, Center for Prostate Cancer, National Cancer Center, 323 Ilsan-ro, Ilsandong-gu, Goyang-si, Gyeonggi-do 10408, Republic of Korea. ${ }^{2}$ Biometrics Research Branch, Division of Cancer Epidemiology and Prevention, Research Institute and Hospital of National Cancer Center, Goyang, South Korea.

Received: 31 July 2017 Accepted: 2 July 2019

Published online: 15 July 2019

\section{References}

1. Calvo E, Schmidinger M, Heng DY, Grünwald V, Escudier B. Improvement in survival end points of patients with metastatic renal cell carcinoma through sequential targeted therapy. Cancer Treat Rev. 2016;50:109-17.

2. Pal SK, Nelson RA, Vogelzang N. Disease-specific survival in de novo metastatic renal cell carcinoma in the cytokine and targeted therapy era. PLoS One. 2013;8:e63341.

3. Bedke J, Gauler T, Grünwald V, Hegele A, Herrmann E, Hinz S, et al. Systemic therapy in metastatic renal cell carcinoma. World J Urol. 2017;35:179-88.

4. Takagi T, Kondo T, Kennoki T, lizuka J, Kobayashi H, Hashimoto Y, et al. Comparison of survival rates in patients with metastatic renal cell carcinoma according to treatment era including cytokine and targeted therapy. Jpn J Clin Oncol. 2013;43:439-43.

5. Fisher RI, Rosenberg SA, Fyfe G. Long-term survival update for high-dose recombinant interleukin-2 in patients with renal cell carcinoma. Cancer J Sci Am. 2000;6(Suppl 1):S55-7.

6. Dabestani S, Marconi L, Bex A. Metastasis therapies for renal cancer. Curr Opin Urol. 2016;26:566-72.

7. Kim SH, Park WS, Kim SH, Joung JY, Seo HK, Lee KH, et al. Systemic treatments for metastatic renal cell carcinoma: 10-year experience of immunotherapy and targeted therapy. Cancer Res Treat. 2016;48:1092-101.

8. Heng DY, Xie W, Regan MM, Warren MA, Golshayan AR, Sahi C, et al. Prognostic factors for overall survival in patients with metastatic renal cell carcinoma treated with vascular endothelial growth factor-targeted agents: results from a large, multicenter study. J Clin Oncol. 2009;27:5794-9.

9. Motzer RJ, Bacik J, Schwartz LH, Reuter V, Russo P, Marion S, et al. Prognostic factors for survival in previously treated patients with metastatic renal cell carcinoma. J Clin Oncol. 2004;22:454-63.

10. Kim SH, Kim S, Joo J, Seo HK, Joung JY, Lee KH, et al. A retrospective study of predictive factors for unexpectedly prolonged or shortened progression-free survival and overall survival among patients with metastatic renal cell carcinoma who received first-line targeted therapy. BMC Cancer. 2016;16:577.

11. Eisenhauer EA, Therasse P, Bogaerts J, Schwartz LH, Sargent D, Ford R, et al. New response evaluation criteria in solid tumours: revised RECIST guideline (version 1.1). Eur J Cancer. 2009;45:228-47.

12. Moch $\mathrm{H}$. The WHO/ISUP grading system for renal carcinoma. Pathologe. 2016;37:355-60.

13. Moch H, Artibani W, Delahunt B, Ficarra V, Knuechel R, Montorsi F, et al. Reassessing the current UICC/AJCC TNM staging for renal cell carcinoma. Eur Urol. 2009;56:636-43.

14. Kim SH, Park WS, Park EY, Park B, Joo J, Joung JY, et al. The correlation of tissue-based biomarkers in primary and metastatic renal cell carcinoma lesions: a tissue microarray study. Korean J Urol Oncol. 2016;14:152-8.
15. Motzer RJ, Mazumdar M, Bacik J, Berg W, Amsterdam A, Ferrara J. Survival and prognostic stratification of 670 patients with advanced renal cell carcinoma. J Clin Oncol. 1999;17:2530-40.

16. Naito S, Yamamoto N, Takayama T, Muramoto M, Shinohara N, Nishiyama K, et al. Prognosis of Japanese metastatic renal cell carcinoma patients in the cytokine era: a cooperative group report of 1463 patients. Eur Urol. 2010;57: $317-25$.

17. Wada Y, Takahashi W, Kawano Y, Eto M. Current status of pharmacotherapy against metastatic renal cell carcinoma in Japan. Int J Urol. 2012;19:284-95.

18. Abe H, Kamai T. Recent advances in the treatment of metastatic renal cell carcinoma. Int J Urol. 2013;20:944-55.

19. Kammerer-Jacquet SF, Brunot A, Pladys A, Bouzille G, Dagher J, Medane S, et al. Synchronous metastatic clear-cell renal cell carcinoma: a distinct morphologic, immunohistochemical, and molecular phenotype. Clin Genitourin Cancer. 2017;15:e1-17.

20. Peters I, Hora M, Herrmann TR, von Klot C, Wegener G, Stansky P, et al. Incidence of synchronous and metachronous adrenal metastases following tumor nephrectomy in renal cell cancer patients: a retrospective bi-center analysis. Springerplus. 2013;2:293.

21. Rajarubendra N, Pook D, Frydenberg M, Appu S. Rare synchronous metastases of renal cell carcinoma. Urol Ann. 2014;6:157-8.

22. Santoni M, Conti A, Partelli S, Porta C, Sternberg CN, Procopio G, et al. Surgical resection does not improve survival in patients with renal metastases to the pancreas in the era of tyrosine kinase inhibitors. Ann Surg Oncol. 2015;22:2094-100.

23. Tilki D, Hu B, Hguyen HG, Dall'Era MA, Bertini R, Carballido JA, et al. Impact of synchronous metastasis distribution on cancer specific survival in renal cell carcinoma after radical nephrectomy with tumor thrombectomy. J Urol. 2015;193:436-42.

24. Zastrow S, Phuong A, von Bar I, Novotny V, Hakenberg OW, Wirth MP. Primary tumor size in renal cell cancer in relation to the occurrence of synchronous metastatic disease. Urol Int. 2014;92:462-7.

25. Ingimarsson JP, Sigurdsson MI, Hardarson S, Petursdottir V, Jonsson E, Einarsson GV, et al. The impact of tumour size on the probability of synchronous metastasis and survival in renal cell carcinoma patients: a population-based study. BMC Urol. 2014;14:72.

26. Bamias A, Tzannis K, Papatsoris A, Oudard S, Beuselinck B, Escudier B, et al. Prognostic significance of cytoreductive nephrectomy in patients with synchronous metastases from renal cell carcinoma treated with first-line sunitinib: a European multiinstitutional study. Clin Genitourin Cancer. 2014; 12:373-83.

\section{Publisher's Note}

Springer Nature remains neutral with regard to jurisdictional claims in published maps and institutional affiliations.

Ready to submit your research? Choose BMC and benefit from:

- fast, convenient online submission

- thorough peer review by experienced researchers in your field

- rapid publication on acceptance

- support for research data, including large and complex data types

- gold Open Access which fosters wider collaboration and increased citations

- maximum visibility for your research: over $100 \mathrm{M}$ website views per year

At BMC, research is always in progress.

Learn more biomedcentral.com/submissions 